\title{
ESTADO DEL CULTIVO EN EL BINOMIO CIUDAD-HUERTA DE VALENCIA POR MEDIO DE APLICACIONES ARTÍSTICAS INTERACTIVAS
}

\author{
María José Gutiérrez González
}

Escuela de Arte y Superior de Diseño de Valencia EASD

\section{Resumen}

Durante siglos ha perdurado una estrecha relación de dependencia y prosperidad entre la ciudad y la huerta en Valencia. En la actualidad, la interacción en dicho binomio se ha ido debilitando progresivamente, ya que el elemento urbano se ha impuesto con sus ampliaciones descontroladas, sumado a un estilo de vida y una producción de riqueza desvinculados de la tierra y el cultivo. Este paisaje con una elevada carga cultural se encuentra en un estado crítico de conservación y todavía hoy no se vislumbra un claro porvenir. La producción artística, a través de aplicaciones interactivas se convierte en un mecanismo para combatir y visibilizar esta problemática. A través de la contraposición de datos del pasado sobre el paisaje que recorre el trayecto de la histórica acequia de la Rascanya. En consecuencia, utilizamos los escritos del botánico Antonio José Cavanilles que desarrolló a finales del siglo XVIII sobre la ciudad y sus alrededores, relatan la relación en equilibrio que permitía la convivencia y la riqueza en ambos espacios, además de un optimizado aprovechamiento de los recursos naturales.

\section{Palabras clave: ACEQUIA RASCANYA (VALENCIA); APLICACIONES ARTÍSTICAS INTERACTIVAS; CULTURA DE LA IRRIGACIÓN; PRODUCCIÓN SOSTENIBLE}

\section{STATE OF THE CROP IN THE CITY-GARDEN BINOMIAL OF VALENCIA THROUGH INTERACTIVE ARTISTIC APPLICATIONS}

\section{Abstract}

For centuries, a close relationship of dependence and prosperity between the town and the vegetable garden in Valencia has lasted. Currently, the interaction in this binomial has been gradually weakening, since the urban element has imposed itself with its uncontrolled expansions, added to a lifestyle and a production of wealth disconnected from land and cultivation. This landscape with a high cultural load is in a critical state of conservation and still today there is no clear future. Artistic production, through interactive applications will become a mechanism to fight and make visible this problem. Through the contrast of past data on the landscape that runs along the route of the historic ditch of the Rascanya. Consequently, we use the writings of the botanist Antonio José Cavanilles that he developed at the end of the 18 century on the town and its surroundings, related the relationship in balance that allowed the coexistence and wealth in both spaces, in addition to an optimized use of natural resources.

Keywords: RASCANYA IRRIGATION CANAL (VALENCIA); INTERACTIVE ARTISTIC APPLICATION; IRRIGATION CULTURE; SUSTAINABLE PRODUCTION

\footnotetext{
Gutiérrez González, María José. 2020. "Estado del cultivo en el binomio ciudad-huerta de Valencia por medio de aplicaciones artísticas interactivas". AusArt 8 (1): 233-245. D0I: 10.1387/ausart.21494
}

\section{AUSART}




\section{INTRODUCCIÓN}

En la segunda década del siglo XXI podemos confirmar que la expansión de los núcleos urbanos continúa en esa dinámica de crecimiento, asimismo durante el transcurso de este siglo se pronostica una ininterrumpida diáspora de la población de los territorios rurales a las vastas urbes. Un abandono de los enclaves agrícolas y el debilitamiento de las pequeñas poblaciones, en beneficio de las metrópolis y sus incesantes ramificaciones. Ante esta situación, muchas ciudades europeas son conscientes del riesgo que supone la desaparición de sus espacios periurbanos y paisajes culturales de gran valor. Aquí nos centramos en la huerta de Valencia, una modalidad de paisaje que durante siglos se ha estructurado en las proximidades de la urbe. Este paisaje con una elevada carga identitaria se encuentra en un estado crítico de conservación y todavía hoy no se vislumbra un claro porvenir, incluso "pocas ciudades europeas disponen de un entorno tan singular $y$, sin embargo, tan desprotegido" (Romero \& Francés 2013, 7). Durante siglos ha subsistido una estrecha relación de prosperidad y dependencia entre la ciudad y la huerta. Pero la interacción de este binomio, en el transcurso del siglo XX y de manera más pronunciada en el siglo $\mathrm{XXI}$, se ha ido difuminando progresivamente, por la imposición de un estilo de vida y una producción de riqueza completamente ajena a la tierra y al cultivo. En la actualidad, tras los últimos vaivenes económicos del mundo globalizado, con su ritmo acelerado de inversiones especulativas al margen del territorio, ha transcendido en la ciudadanía, de tal modo, se ha gestado un activismo en contra del avance urbano descontrolado, y en defensa de la preservación de la memoria colectiva construida durante siglos en este singular espacio rural. "El paisaje es construcción cultural del territorio" (Maderuelo 2008, 280). Ante este clima de protesta, se hace más urgente una intervención de políticas públicas para garantizar la actividad agraria, y así obtener una rentabilidad en las producciones similares a cualquier otro sector en la sociedad. Al mismo tiempo, podríamos esquivar la tentación de transformar este paisaje en un mero parque temático de consumo turístico. "El problema fundamental de viabilidad de la Huerta de Valencia y del conjunto de los regadíos históricos radica en la preservación de la actividad agrícola" (Romero \& Francés 2013, 10). En definitiva, mantener la producción en la huerta metropolitana conlleva unas directrices de crecimiento urbanístico adaptadas a este contexto cultural específico. Donde las nuevas ampliaciones se desarrollen en convivencia y complementariedad con las características naturales y las capas sedimentadas de la intervención humana en el territorio. “(...) volver de nuevo la mirada al territorio, (...) buscando su utilidad en armonía con la naturaleza y creando belleza" (Maderuelo 2010, 233). 
La crisis ecológica y ambiental empezó a tener notoriedad durante la década de los años 70 del siglo pasado, y hoy en día representa una seria amenaza para el porvenir de las próximas generaciones. El paradigma de presente perpetuo impuesto (Jameson), nos impide detenernos y reflexionar sobre el verdadero impacto que puede tener este constante deterioro a largo plazo. Ante este panorama, queda todavía más justificado el posicionamiento de proteger el paisaje de la huerta, ya que contribuiría al bienestar de la salud de los valencianos, puesto que favorece a un estilo de vida más saludable, la revalorización de la agricultura hortícola próxima a la ciudad y respetuosa con el medio ambiente, "calidad, diversidad y connotaciones culturales asociadas a las prácticas gastronómicas son valores en alza" (Calatayud 2005, 161). El paisaje de la huerta debería resultar bastante atractiva para la construcción de la nueva ciudad metropolitana, como mencionaba Juan Miguel del Rey (2002). Un paisaje considerado en otras épocas como un majestuoso jardín en su conjunto, así podemos hallarlo descrito en los poemas árabes de los siglos XI y XII, como también en las observaciones de la obra del ilustre botánico Antonio José Cavanilles, a finales del siglo XVIII. Es muy probable que nos encontremos en la última oportunidad por salvaguardar estas parcelas de cultivo, junto a su valiosa cultura, porque tras la considerable reducción que han sufrido en las últimas décadas, simplemente quedaría la alternativa de rescatar intencionadamente y desde las instituciones públicas la histórica relación, en caso contrario, la tendencia será su abandono y definitiva desaparición.

Los paisajes de huertas y vegas en entornos urbanos, modelados por agriculturas de viejos regadíos y característicos sistemas de asentamiento asociados, suscitan hoy un interés especial por sus valores agroecológicos, productivos, hidráulicos y etnográficos, pero también por el papel ambiental que desempeñan o deberían desempeñar en contextos territoriales crecientemente saturados.

(Maderuelo 2010, 39)

Binomio armónico que tuvo su fundación en el período andalusí, aunque hubo unas aportaciones precedentes en la época romana, no fue hasta el siglo $X$ cuando se desarrolló la compleja trama de irrigación para la implantación de un sistema de regadío en estas tierras, y que posteriormente, ante tal espectacular riqueza desarrollada, los pobladores cristianos continuaron con estos saberes hidráulicos en el siglo XIII. Un complejo entramado que facilitaba la distribución del agua a un número infinito de parcelas. Un tejido que fue perfeccionándose con el tiempo, pues se fueron creando canales principales para ampliar y organizar las zonas de cultivo en demanda de las necesidades de la creciente población de la ciudad. Paisaje que se mantuvo prácticamente en 
su totalidad hasta entrado el siglo XX, ya que "la ciudad hizo a la huerta y la huerta hizo a la ciudad, al favorecer su crecimiento demográfico y económico" (Romero \& Francés 2013, 39), desde entonces ha sido un progresivo desamparo de la huerta hasta nuestros días. Como mencionaba Salvador Calatayud "la ciudad ha vencido y ello, en buena medida, porque ya no necesita a la huerta" $(2005,158)$.

En conclusión, una sabiduría del dominio del agua acumulada por el esfuerzo de los valencianos a lo largo de diversos siglos, que su pérdida supondría, en este siglo de emergencia climática, un error intolerable para este territorio.

\section{LA PRODUCCIÓN ARTÍSTICA COMO MECANISMO}

La producción artística simboliza una alternativa combatiente al servicio de la sociedad ante cualquier tipo de imposición. Por consiguiente, el arte también puede ayudar a la orientación de los procesos de rehabilitación del territorio, "la función social del arte y la implicación del artista en los procesos de transformación y, en concreto, sobre la necesidad de continuar insuflando energía, desde el ámbito del arte, para evitar la banalización del territorio" (Maderuelo 2008, 285). En la actualidad, existen muchas propuestas cartográficas con una visión experimental que exploran otros caminos para acceder y conocer el espacio habitado, acompañados de reivindicaciones colectivas, divulgaciones de injusticias. Aquí, nos centramos en los daños causados en el paisaje. A continuación, presentamos un tipo de cartografía artística formalizada en aplicaciones interactivas que intentan revelar al usuario el grave estado de la identidad del paisaje de la huerta. La riqueza de un cultivo milenario que ha sido destruido intencionadamente en las últimas décadas y que entrañaría una pieza significativa para entender este territorio.

\subsection{Aplicación interactiva Identidad _ Cavanilles}

La primera aplicación interactiva presentada en esta publicación se denominó Id_Cavanilles, en la cual nos planteamos trabajar a partir de 10 textos extraídos de la obra del botánico valenciano, Antonio José Cavanilles Palop. Nos hemos basado en la visionaria obra Observaciones sobre la historia natural 
del Reino de Valencia y en especial, en el Tomo I de 1795. Esta obra supuso una rica aportación de investigación, ya que hizo un análisis muy variado y completo de las tierras del reino de Valencia. Este proyecto lo emprendió en 1791 y estuvo compuesto por varias expediciones, en cada uno de dichos viajes, el autor abordaba distintas zonas del reino valenciano. Exploraciones basadas en un lento y arduo trabajo de campo, pues le ocupaban largos períodos de meses. Estas tareas las intercalaba con estancias de descanso en Madrid, donde aprovechaba para recopilar toda la información recogida. De tal manera, que la mencionada obra tardó unos años hasta finalizarla a finales del siglo XVIII. La obra de Cavanilles sobre el reino de Valencia tiene un mérito incuestionable, ya no solamente por su aportación botánica, junto con sus correcciones y ampliaciones geográficas sobre el reino, las cuales fueron muy valiosas, sino además por su visión holística de estas tierras y sus gentes. La obra contiene datos etnográficos, económicos, sanitarios, e incluso nos atreveríamos a decir, aportaciones de carácter 'ecológico', aunque el término no existía en esa época.

Hemos seleccionado el Tomo I, pues es aquí donde el autor expuso toda la investigación realizada en la ciudad de Valencia y de sus poblaciones próximas. Así pues, nos ofrecía la posibilidad de recopilar las observaciones que hizo Cavanilles sobre nuestra zona de estudio, es decir, el paisaje que recorre la acequia de la Rascanya, tanto en la zona de l'horta nord, junto con las poblaciones de Alboraia y Tavernes Blanques, como también, las menciones que hizo sobre el río Turia y sus distribuciones en distintos canales, la zona de Campanar, junto con sus pedanías próximas. Sumado al análisis del aspecto de la entrada principal de la ciudad, la actual calle de Sagunto cercana al parque de Marxalenes.

Los 10 textos han sido mostrados en su escritura original, sin ningún tipo de modificación y adaptación, pues hemos

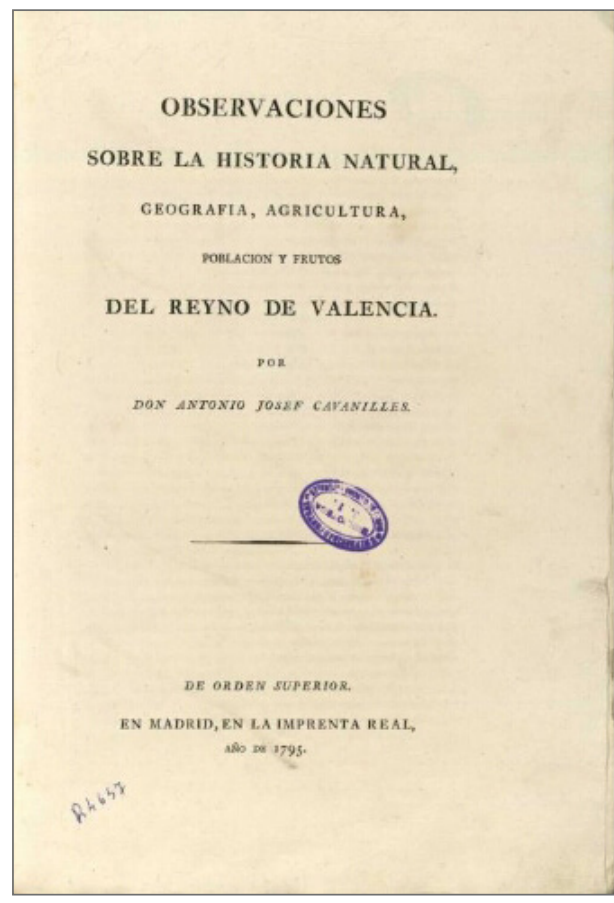

Fig. 1. Página interior de la obra de Antonio José Cavanilles, Imagen digital de la Biblioteca Valenciana 
evaluado que, a pesar de conservar un estilo y una gramática de otra época, su rápida interpretación era bastante óptima, y al mismo tiempo, conservábamos la autenticidad de los escritos.

Por otro lado, continuamos con la misma estrategia en nuestra investigación doctoral, de contraponer datos del pasado con su estado actual, mecanismo que utilizamos para reflexionar sobre la calidad e identidad de nuestro paisaje. En consecuencia, junto a los textos de Cavanilles de finales del siglo XVIII, hemos acompañado unos vídeos grabados en la actualidad, sobre las mismas zonas de huerta y cultivo, por donde discurre nuestra acequia.

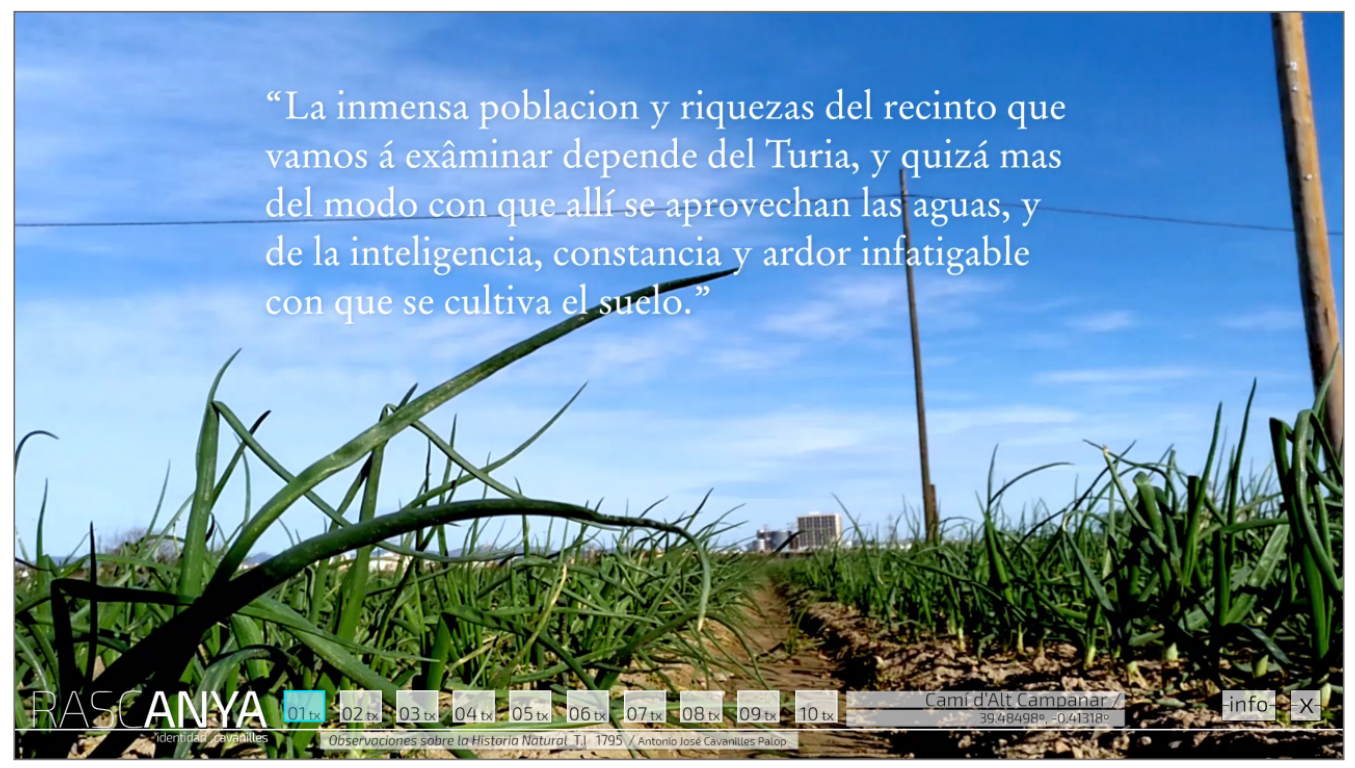

Fig. 2. Interfaz de la aplicación interactiva Identidad_Cavanilles. Imagen de la autora, 2020. https://www.cartografiarascanya.com/identidad_cavanilles/

El acceso a la aplicación se ha solucionado en dos escenas, de tal modo, la primera escena se le ha dotado de un tratamiento introductorio del contenido que posteriormente se muestra, y al mismo tiempo, hemos añadido información asociada con la usabilidad interactiva de la aplicación. Tras la lectura de esta documentación, la escena facilitaba el paso a la siguiente escena principal por medio de la activación de un botón. Esta segunda escena estaba estructurada con un menú principal, ubicado en la parte inferior de la interfaz, el cual contenía 10 botones que habilitaban la navegación entre los distintos textos mencionados. La activación de cada uno de estos botones reproducía 
la animación y visualización de un texto específico, acompañado con un vídeo de cultivo de fondo. En cada reproducción de vídeo además se adjuntaba los datos de localización, en concreto, el nombre de la calle o vía, junto con los datos de latitud y longitud.

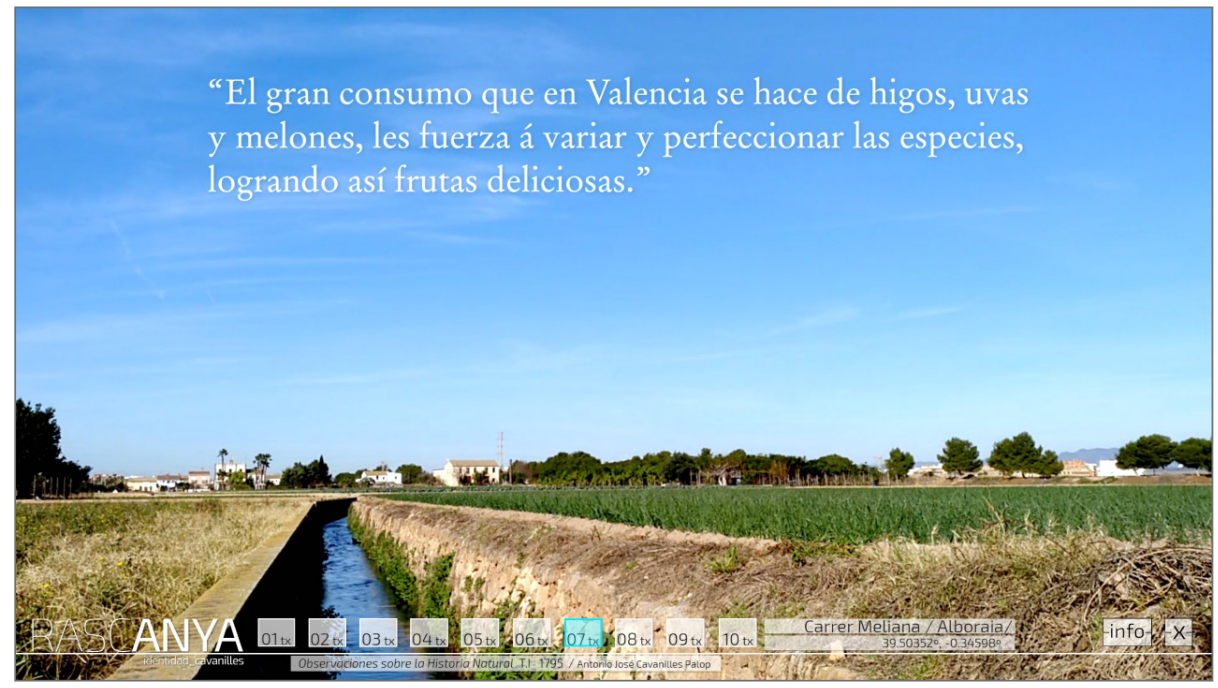

Fig. 3. Interfaz de la aplicación interactiva Identidad Cavanilles. Imagen de la autora, 2020. https://www.cartografiarascanya.com/identidad_cavanilles/

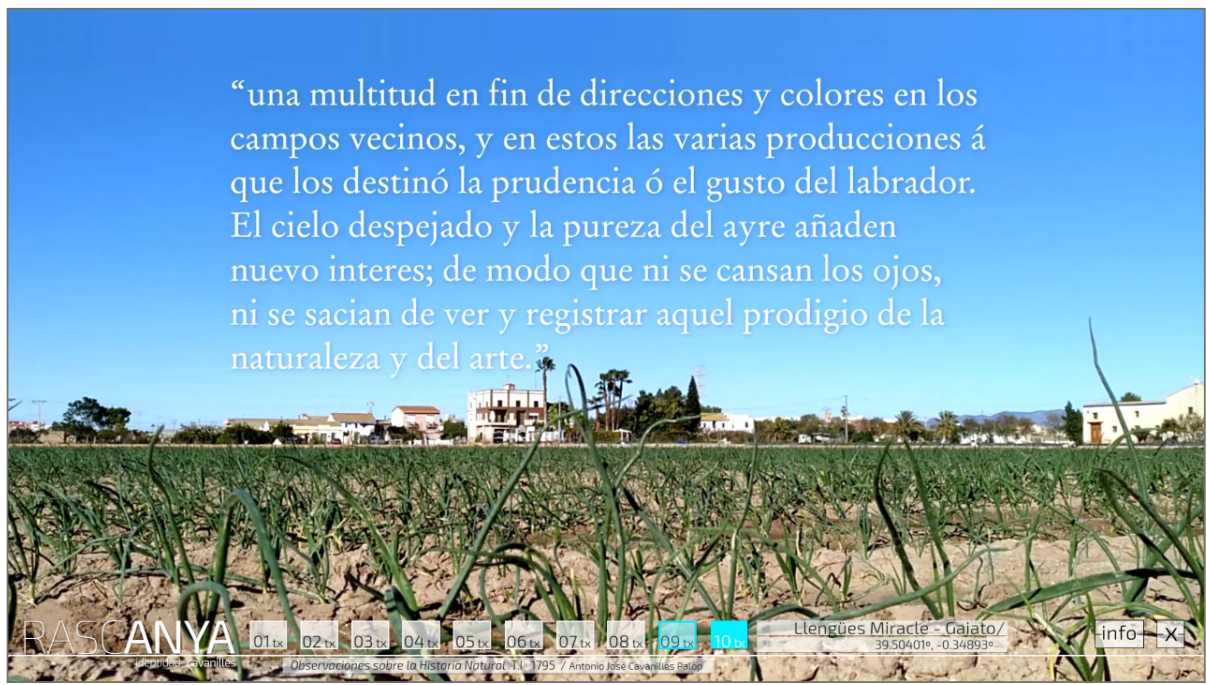

Fig. 4. Interfaz de la aplicación interactiva Identidad_Cavanilles. Imagen de la autora, 2020. https://www.cartografiarascanya.com/identidad_cavanilles/ 
Por último, en el lateral derecho se diseñó una segunda botonera secundaria, con los botones pensados para salir de la aplicación, y para volver a la primera escena introductoria, nombrado info, por si el usuario necesitaba repasar alguna información.

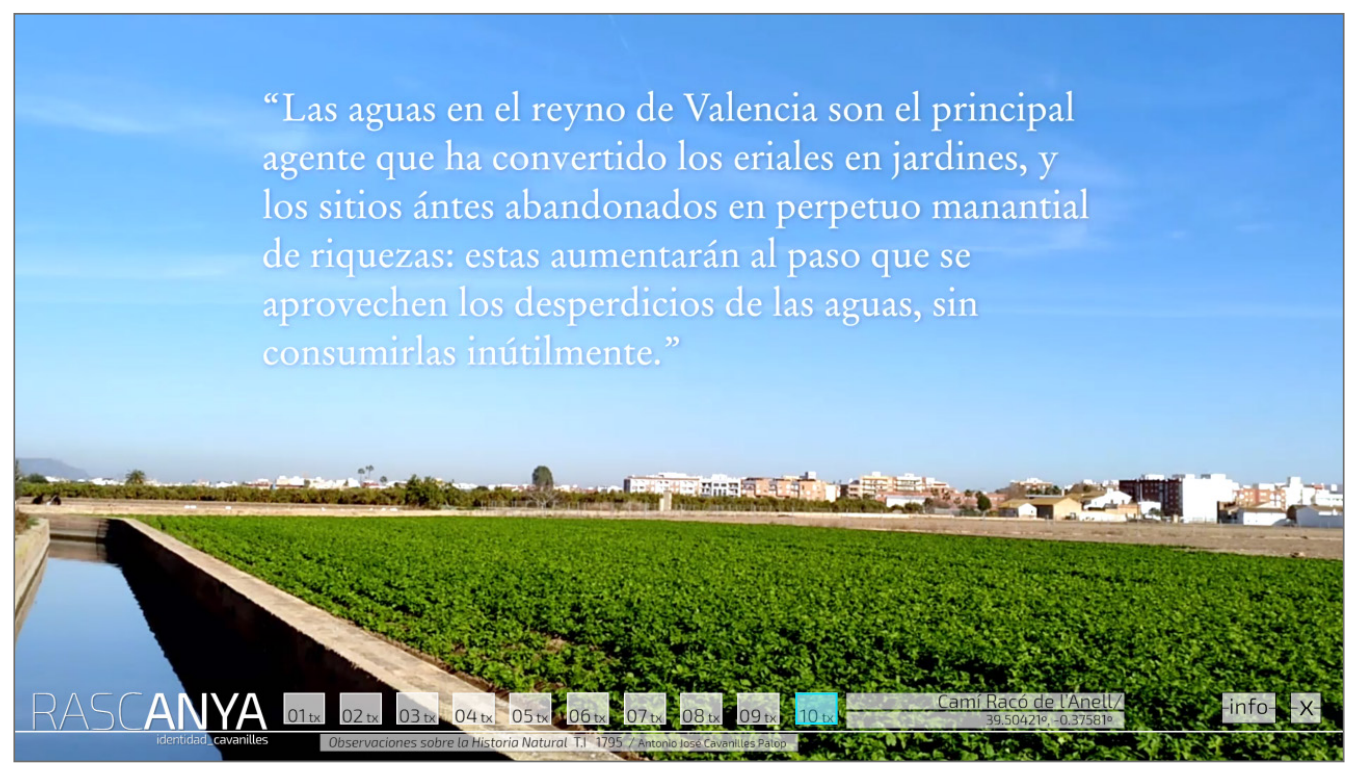

Fig. 5. Interfaz de la aplicación interactiva Identidad_Cavanilles. Imagen de la autora, 2020. https://www.cartografiarascanya.com/identidad_cavanilles/

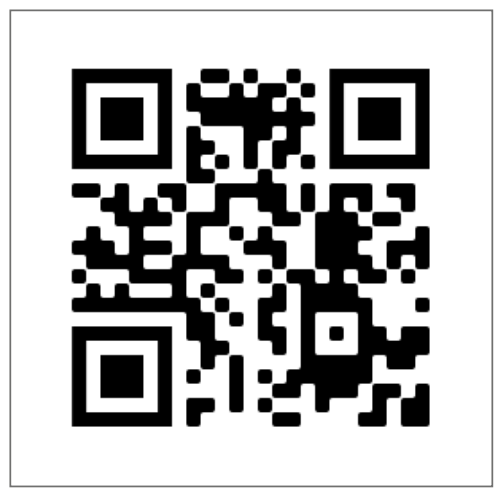

Fig. 6. Código QR que enlaza con un vídeo de simulación de la aplicación interactiva /dentidad_Cultivo. https://vimeo. com/390193221

\subsection{Aplicación interactiva IDENTIDAD - Cultivo}

En relación, a la segunda aplicación denominada Id_Cultivo, que acompañamos en este escrito, debemos mencionar que nuevamente utilizamos la visualización de 10 textos extraídos de la misma obra del botánico Antonio José Cavanilles Palop. Pero en este caso, el criterio de selección de los textos giraba en función a la información y documentación relacionada con el tipo y cantidad de cultivo, estado y calidad de la tierra, productividad y otras funcionalidades asignadas a partir de la estrecha relación con la ciudad de Valencia. De la misma manera, que en la anterior aplicación hemos mantenido 
la escritura original, por su fácil compresión. Únicamente hemos añadido una pequeña leyenda aclarativa, para asegurarnos de su correcta interpretación, en concreto, en la parte inferior de la interfaz, y en relación a una cantidad a modo de abreviatura de la época.

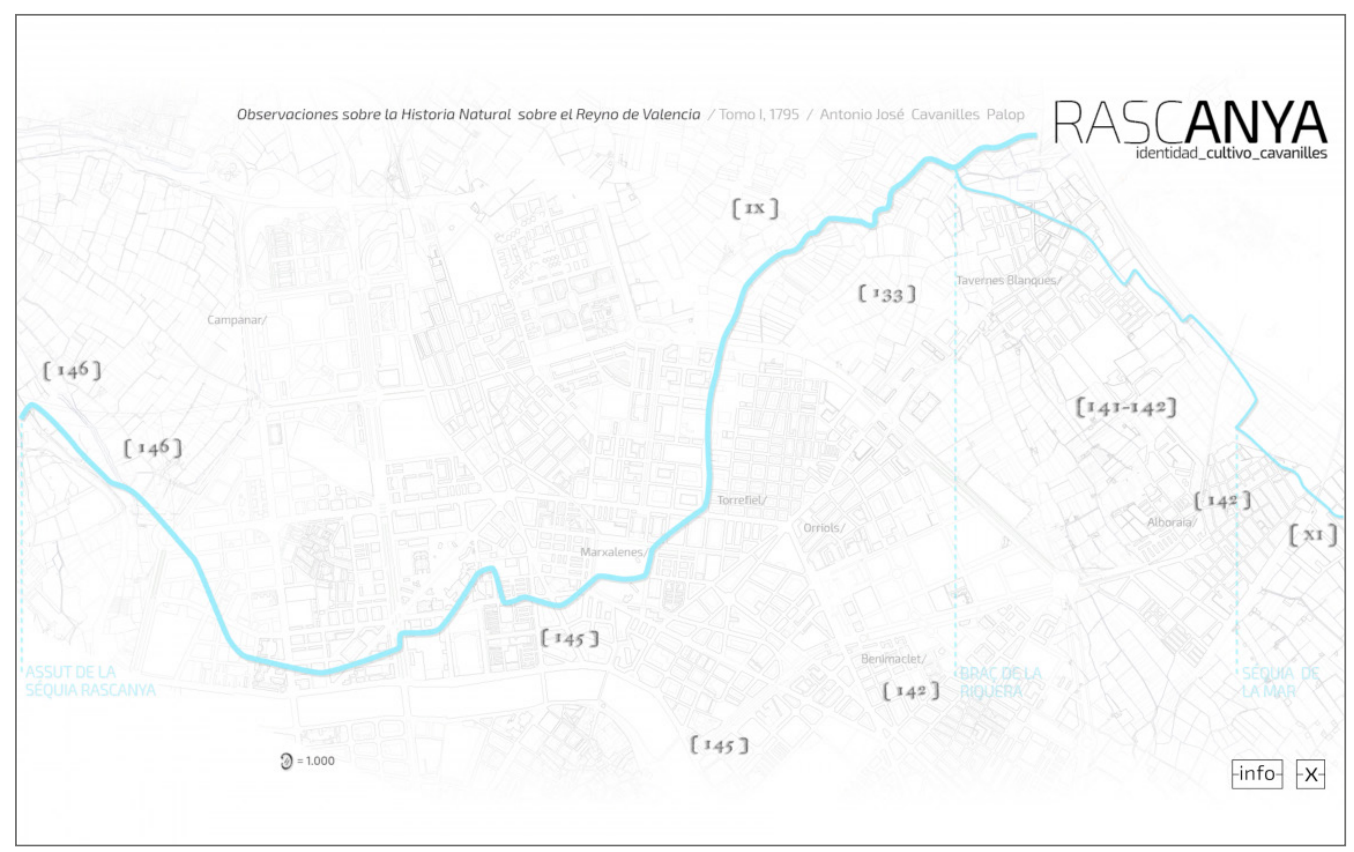

Fig. 7. Interfaz de la aplicación interactiva Identidad_Cultivo Cavanilles. Imagen de la autora, 2020. https://www.cartografiarascanya.com/identidad_cultivo-cavanilles/

Asimismo, la aplicación quedó estructurada con una primera escena introductoria, donde el usuario podía documentarse sobre el contenido que después iba a visualizar, junto también con las pautas informativas asociadas al modo de interacción. A partir de este nivel, y tras la activación de un botón que permitía el acceso a una segunda escena de carácter principal, la cual adoptó el diseño de un mapa callejero de la zona de interés de la ciudad de Valencia, junto con la gráfica azul correspondiente al tramo del recorrido de la acequia, como fondo de la interfaz. Después decidimos incorporar, como medida de apoyo, el nombre de los distintos distritos por donde transitaba la acequia; Campanar, Marxalenes, Benimaclet, Torrefiel, Orriols, y de las localidades de Tavernes Blanques y Alboraia.

Por otro lado, en esta segunda aplicación, el menú principal se formalizó con unos botones con aspecto de cifras de números, que hacían referencia al 
número de las páginas correspondientes de cada texto extraído del Tomo I del libro de Cavanilles. Además, estos botones se dispusieron en los enclaves específicos donde el autor desarrolló sus observaciones de la zona. Así pues, la activación de cada uno de estos botones suponía la reproducción de una animación que visualizaba el texto correspondiente. En esta aplicación hemos intentado conservar la apariencia original de los textos obtenidos del libro, por medio de la edición en un software de posproducción de imagen digital. Para finalizar, en la parte inferior de la interfaz, en concreto, en el lateral derecho, también se dispuso una segunda botonera secundaria, con los botones de salida y de info, como en el resto de las aplicaciones.

Las dos aplicaciones interactivas presentadas fueron resueltas con el software Unity $3 d$, con un diseño de interfaz bidimensional y una resolución de pantalla de 1920 × 1080 píxeles. Aunque hay que decir, que dichas aplicaciones pueden visualizarse en otras resoluciones más pequeñas, de tal modo, que pueden ejecutarse en dispositivos como, smartphones o tablets, sin ningún problema de legibilidad.

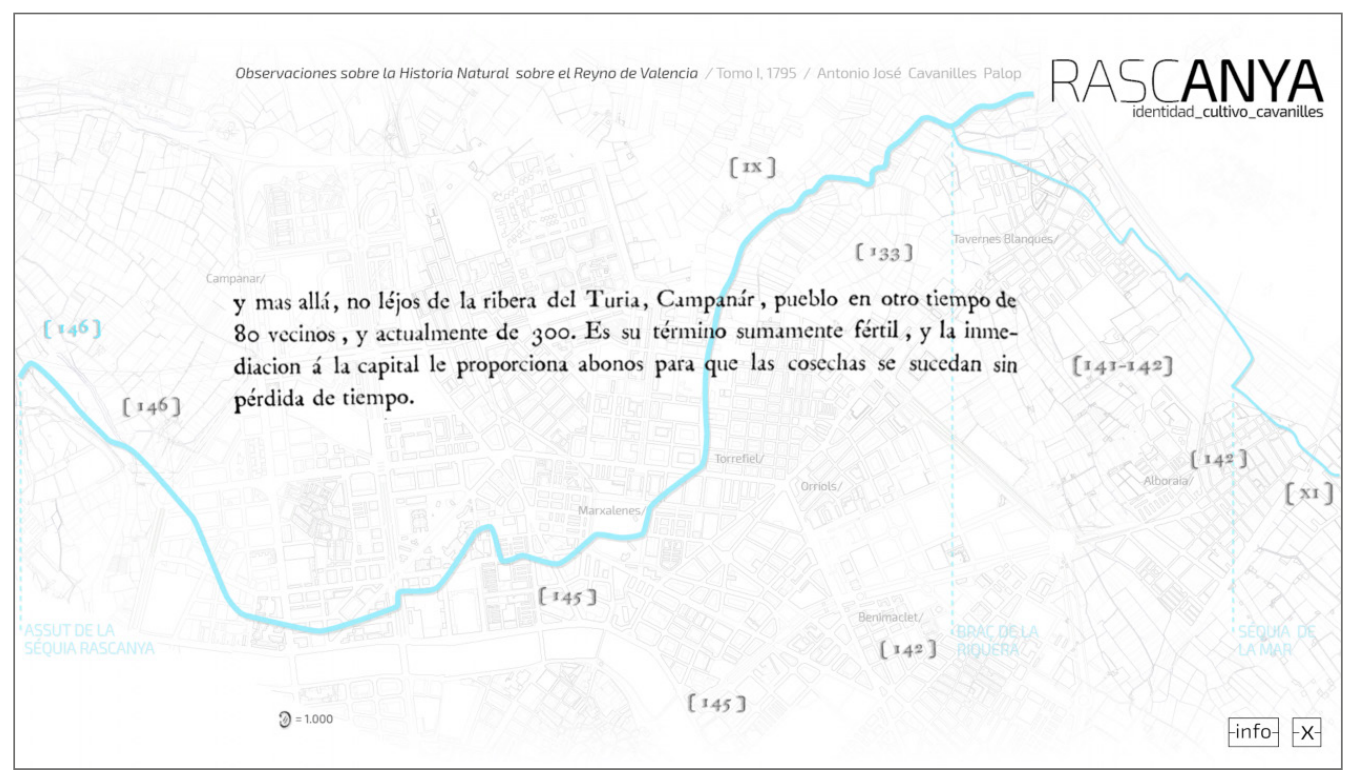

Fig. 8. Interfaz de la aplicación interactiva Identidad_Cultivo Cavanilles. Imagen de la autora, 2020. https://www.cartografiarascanya.com/identidad_cultivo-cavanilles/ 


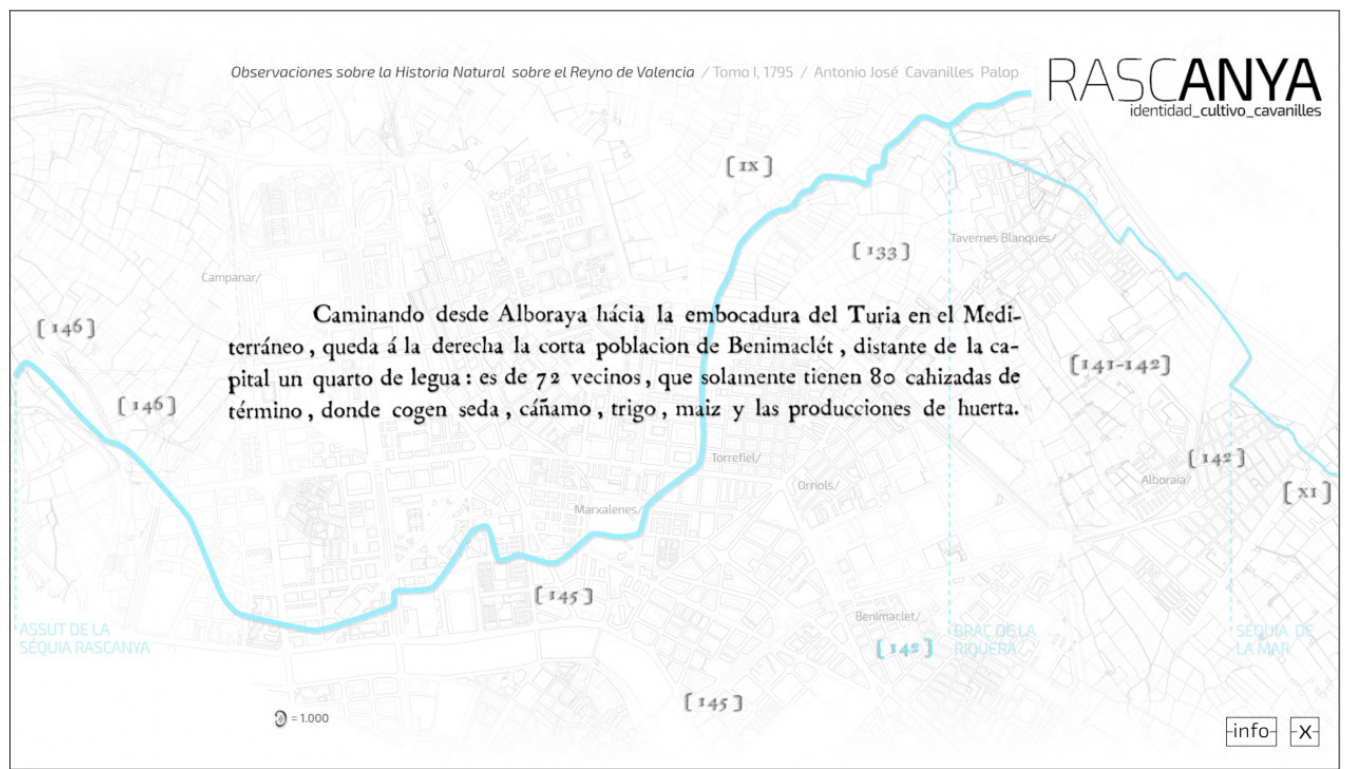

Fig. 9. Interfaz de la aplicación interactiva Identidad_Cultivo Cavanilles. Imagen de la autora, 2020. https://www.cartografiarascanya.com/identidad_cultivo-cavanilles/

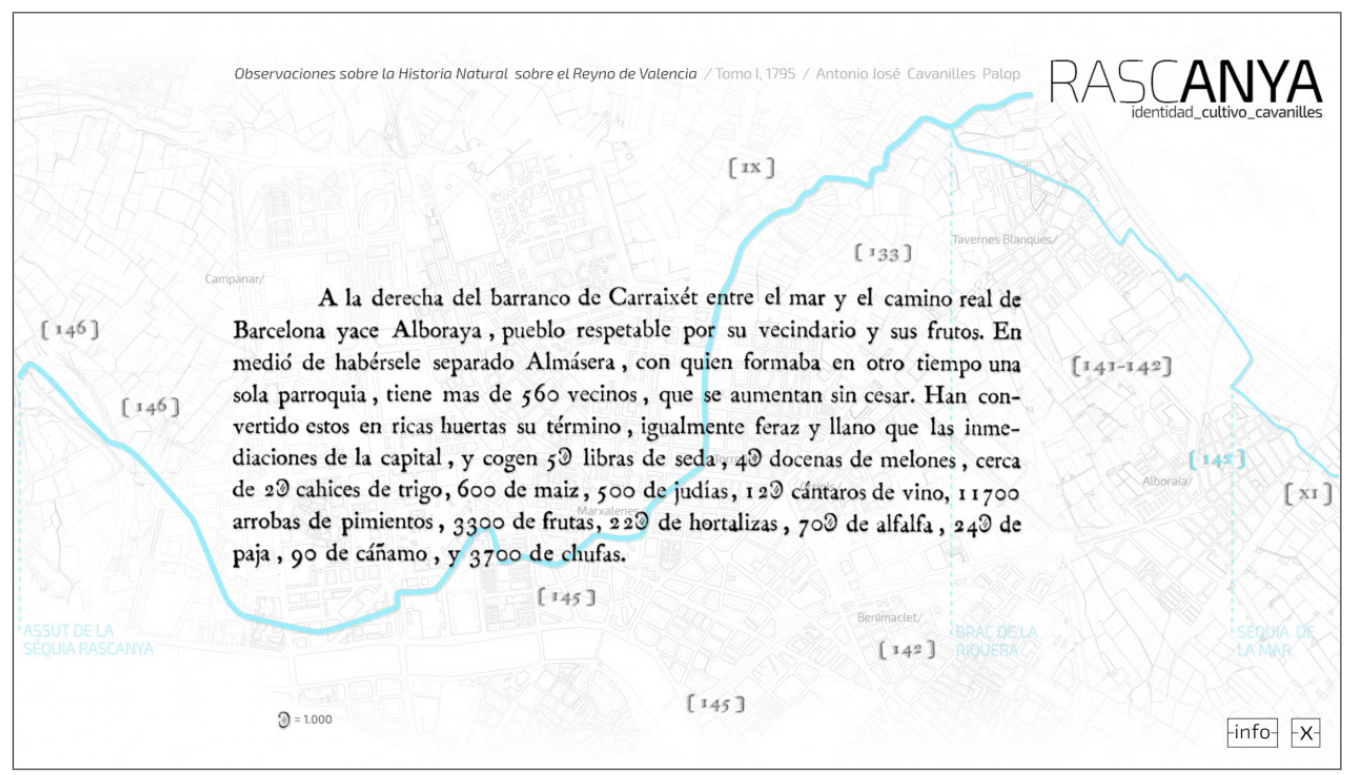

Fig. 10. Interfaz de la aplicación interactiva Identidad_Cultivo Cavanilles. Imagen de la autora, 2020. https://www.cartografiarascanya.com/identidad_cultivo-cavanilles/ 


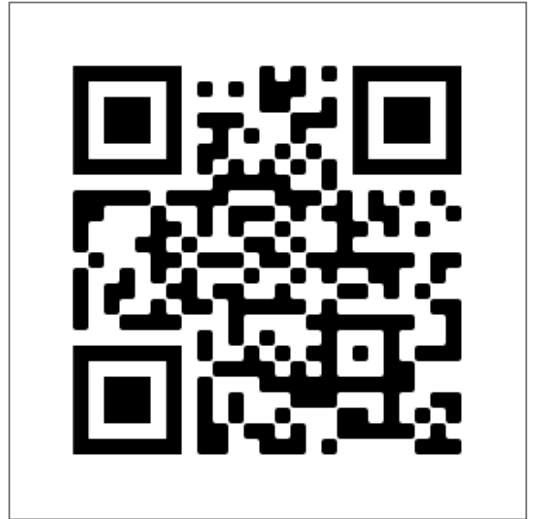

Fig. 11. Código QR que enlaza con un vídeo de simulación de la aplicación interactiva Identidad_Cultivo.

https://vimeo.com/387649637
Para finalizar, hacer mención del motivo de la muestra de estos escritos del siglo XVIII, no han tenido una intención ni mirada nostálgica de una época pasada mejor. En realidad, la visibilización en nuestra contemporaneidad a través de las aplicaciones tiene el objetivo de rescatar la actitud de aprovechamiento y productividad de estas ricas tierras de cultivo, junto con la relación equilibrada y saludable que se generaba entre la huerta y la ciudad, binomio cultural y tan singular de este territorio, que ha sido roto en las últimas décadas y que deberíamos revisar, ante el estado actual de abandono en el que se encuentra este paisaje.

\section{CONCLUSIONES}

La sociedad del siglo XXI saturada de información, donde proliferan grandes cantidades de datos capaces de configurar una realidad paralela al margen de las dimensiones espacio-temporales, las cuales habrían quedado reducidas, optimizadas e ignoradas paulatinamente. Se añadiría la inclinación, de una constante actualización casi al instante de dicha información, esta renovación infinita del momento presente conlleva el olvido de elementos y capas de cualquier otro tiempo. Nos encamina a la ausencia de perspectiva en la reflexión y por extensión a la supresión de la memoria colectiva y una cultura erigida a lo largo del tiempo. En este punto aparece el paisaje, concepto protagonista en este artículo, ya que hemos defendido la urgencia de su protección ante las dinámicas imperantes de habitar. "El paisaje posee significados naturales e históricos. Pero también posee otros que remiten a referencias culturales y sociales, en sus identificaciones, en su personalidad, en sus valores. Es un espacio común de la vida colectiva de un pueblo" (Romero \& Francés 2013, 11).

Por otro lado, nos hallamos en un período de catástrofes climáticas cada vez más frecuentes, junto con unos índices de contaminación en el planeta que resultan alarmantes y que reclaman una intervención más rigurosa. Por consiguiente, el estilo de vida dominante, de carácter globalizado estaría siendo 
cuestionado en nuestros días, así pues, reivindicamos la búsqueda de otras opciones de habitar, con una relación más próxima con las características e historia de un territorio. Aquí, la producción artística debe adoptar un papel responsable y comprometido con este tipo de necesidades sociales. El combate con el discurso oficial a través de la exposición de otras realidades. En este caso, hemos presentado unas aplicaciones interactivas que revelaban un tipo de datos de otro tiempo, los cuales en nuestra contemporaneidad suelen ser relegados y, por el contrario, pensamos que podrían ser de una gran utilidad para la reconfiguración y la transformación que exige el territorio. Datos del cultivo con su cultura de la irrigación construida a lo largo de varios siglos, que deberían tener un papel determinante para las nuevas expansiones urbanas. "Si redescubrimos la continuidad del agua, podremos recuperar la continuidad ecológica de los drenajes del territorio y habremos puesto la primera piedra para obtener las continuidades cívicas que nuestra metrópoli requiere" (Maderuelo 2008, 312).

El territorio de la ciudad de Valencia y sus proximidades contiene un entramado histórico de un gran valor, caminos de agua que podrían articular las renovadas extensiones urbanas en complementariedad y con un aprovechamiento sostenible de los elementos rurales.

\section{Referencias bibliográficas}

Cavanilles y Palop, Antonio José. 1797. Observaciones sobre la historia natural, geografía, agricultura, población y frutos del Reyno de Valencia. Madrid: Imprenta Real

Calatayud Giner, Salvador. 2005. "La ciudad y la huerta”. Historia Agraria 35: 145-64

Jameson, Frederic. 1999. "Posmodernismo y sociedad de consumo". En El giro cultural: Escritos seleccionados sobre el posmodernismo 1983-1998, traducción, Horacio Pons. Buenos Aires: Manantial.

López Cuenca, Rogelio. 2015. Radical geographics. València: IVAM

Maderuelo Raso, Javier, dir. 2007. Paisaje y arte. Paolo Bürgi et al.; epílogo, Alberto Carneiro \& Jesús Mari Lazcano. Madrid: Abada

Maderuelo Raso, Javier, dir. 2008. Paisaje y territorio. Antonio Ansón et al. Madrid: Abada

Maderuelo Raso, Javier, dir. 2010. Paisaje y patrimonio. Diego Arribas et al. Madrid: Abada

Rey Aynat, Miguel del. 2002. Alqueries: Paisatge i arquitectura en I'horta. Amb la collaboració de Vicenç Rosselló et al. València: Consell Valencià de Cultura

Romero González, Joan \& Miquel Frances Domenech, eds. 2013. La Huerta de Valencia: Un paisaje cultural con futuro incierto. Valencia: Universitat de València 